\title{
RELAÇÃO DO TEMPO DE USO DE ANTICONCEPCIONAIS HORMONAIS ORAIS E TEMPO PARA CONCEBER
}

\section{RSPSP-141}

Ciari $\mathrm{J}_{\mathrm{R}, \text {, }}$. et al. - Relacão do tempo de uso de anticoncepcionais hormo nais orais e tempo para conceber. Rev. Saúde públ., S. Paulo, 6: 273-81, 1972.

Resumo: Observou-se um total de 565 mulheres pareadas segundo idade, nivel sócio-economico e estado de saúde, divididas em quatro grupos. Um grupo controle com 155 mulheres e três 'grupos de estudo sendo: grupo I com 162 mulheres que tomaram anticoncepcional hormonal oral durante um ano; grupo II com 127 mulheres que tomaram o mesmo anti. concepcional por dois anos e o grupo III, com 121 mulheres, repetiu as mesmas condiçōes por três anos. Todas tomaram o mesmo anticoncepcional sob a mesma forma. Feitas as curvas de frequiência relacionando tempo de uso com tempo para conceber, verificou-se uma significativa diferença entre as curvas dos grupos controle e I em rclação aos grupos II e III. O material foi submetido $\dot{a}$ análise de variância que confirmou os resultados das curvas. Conclui-se que o uso de anticoncepcionats normonats orais, a partir do segundo ano, interfere com o sistema reprodutor determinando um aumento de prazo para conceber em relação aos grupos controle e de um ano de uso.

UnITERmos: Fertilidade ; Anticoncepcionais orais*.

\author{
Cyro CIARI Jr. * \\ Jair L. F. SANTOS ** \\ Eucides Ayres de CASTILHO ***
}

\section{N T RO D U C A O}

Uma das preocupações dos que estudam os anticoncepcionais hormonais orais são os efeitos colaterais que podem advir de seu uso. Desde as mais simples manifestações como ansiedade, náuseas, etc., até sérias complicações vasculares cerebrais, tem sido atribuidas a estas substâncias chamando a atenção de clínicos e pesquisadores.

Tem sido evidenciado, nos casos de patologia da ovulação, o efeito benéfico destes hormônios de síntese para determinar não só a regularização do ciclo menstrual como também favorecer a ovulação que não está se processando ${ }^{2}$. $O$ que não se tem avaliado é quanto eles interferem com a fecundação, segundo o tempo de uso, quando administrado em mulheres com ciclo menstrual normal.

Os autores têm ressaltado que a ação destes anticoncepcionais se faz determinando o bloqueio da ovulação, seja atuando para alguns ao nível do ovário seja, para outros, inibindo relativamente a hipófise ${ }^{4}$. De qualquer forma eles ge. ram na mulher um ciclo artificial onde

* Da Disciplina de Higiene Materna do Departamento de Prática de Saúde Pública da Faculdađe de Saúde Pública da USP - Av. Dr. Arnaldo, 715 - Sáo Paulo, S.P., Brasil.

* Do Centro de Fstudos de Dinamica Populacional da Faculdade de Saude Pública da USP - Av. Dr. Arnaldo, $715-$ Săo Paulo, S. P., Brasil.

*** Do Departamento de Medicina Preventiva da Faculdade de Medicina da USP - Av. Dr. Arnaldo, 455 - Săo Paulo, S. P., Brasil. 
CIARI Jr., C. et aI. - Relação do tempo de uso de anticoncepcionais hormonais orais e tempo para conceber. Rev. Saúde públ., S. Paulo. 6: 273_81. 1972.

a ovulação é excluída. $O$ que resta saber é se, nestas condições, uma inibição de. morada afeta a reposição do ciclo menstrual normal e consequientemente a ovulação. De qualquer forma, estamos diante de uma interferência no processo biológico da reprodução.

Ao ser suspensa a administração dos anticoncepcionais necessitamos saber quanto eles determinaram de alterações no genital feminino.

Como assinala Corfman ${ }^{1}$, para a análise dos efeitos biológicos dos anticoncepcionais sobre a reprodução, devemos levar em conta seis variáveis:

1. O agente ou agentes especificos usados.

2. A quantidade absoluta de cada agente usado.

3. A proporção de cada agente se mais de um for usado.

4. A via de administração.

5. Duração do uso.

6. Idade da mulher.

Por aí se observa que muitos fatores determinam o grau de influência do anticoncepcional oral sobre a fisiologia da reprodução.

No entanto, a melhor observação que nos leva a concluir pela normalidade funcional é a presença da gestação e, numa forma mais acurada, estudar o tem. po que decorre entre a suspensão de ingestão do anticoncepcional e a fecunda. ção. Este espaço, comparado ao comu. mente existente entre mulheres que não tomaram anticoncepcional, nos dará a justa medida do comportamento do aparelho reprodutor.

Corfman ${ }^{1}$, é um dos únicos a fazer referência sobre este tipo de observação, acreditando que este tempo dispendido para engravidar reflete a integridade da função da hipofise e do ovário.

Em nosso trabalho procuramos levar em consideração os seis fatores apontados por CoRfMan 1, uniformizando-os para todos os grupos em observação, isto é, mantendo o mesmo agente em igual quantidade e proporção e administrado pela via oral. Obtivemos uniformidade nos grupos etários. Com estes elementos fixos estudamos o tempo dispendido para engravidar em função de uso do anticoncepcional. Com isto, procuramos evidenciar a interferência que tem $o$ anticoncepcional sobre o processo da fecundação segundo a duração de seu uso.

\section{MATERIAL E METODOS}

Entre mulheres que desejavam tomar anticoncepcional, procuramos constituir três grupos de mulheres semelhantes quanto a idade e condições sócio-econômicas e de saúde. São pacientes observadas em clínicas particulares de nível só. cio-econômico alto e todas com ciclos menstruais dentro de características normais, sem intercorrências clínicas e com idades de 19 a 25 anos. No começo do estudo, todas iniciaram atividade sexual no casamento.

Constituímos quatro grupos de mulheres:

Grupo I - constituído de 162 mulhe res que iniciaram a tomada de anticoncepcionais no ciclo imediatamente anterior a atividade sexual e permaneceram usando-o até um ano;

Grupo $I I$ - constituído de 127 mulheres nas mesmas condiçōes das anteriores mas tomando o anticoncepcional por dois anos consecutivos;

Grupo III - constituído por $121 \mathrm{mu}-$ lheres onde se repetem as condiçóes anteriores mas cuja tomada de anticoncepcional perdurou por três anos; 
CIARI Jr., C. et al. - Relação do tempo de uso de anticoncepcionais hormonais orais e tempo para conceber. Rev. Saúde públ., S. Paulo, 6: 273.81, 1972.

Grupo controle - constituído por 155 pacientes que iniciaram atividade sexual também no casamento e não usaram anticoncepcional hormonal oral ou nenhum outro. Equivale dizer que desejavam engravidar imediatamente.

Para cumprir os itens mencionados por Corfman 1 exigimos os seguintes requisitos para entrar nos grupos I, II e III;

1. tomada sempre de anticoncepcional composto de estrogeno e progestageno, em associação de noretindrone e mestranol ou noretindrone com etinil estradiol não variando as dosagens na associação;

2. uso constante de dosagens iguais;

3. manutenção dos mesmos agentes nas mesmas proporções;

4. utilização da via oral. As tomadas deviam ser constantes excluindo-se do estudo os casos de interrupção;

5. o tempo de uso correspondente a cada grupo sendo admitida tolerân. cia de 30 dias para mais e para menos.

$\mathrm{Na}$ suposição de ocorrerem casos de esterilidade, independentes da tomada de pílulas, usamos como critério o afastamento da mulher que após seis meses de atividade sexual sem anticoncepcional não engravidou. Obtivemos no grupo I, três casos nestas condições $(1,85 \%$ das estudadas): no grupo II, 2 casos $(1,57 \%)$; no grupo III, 2 casos $(1,65 \%)$ e no grupo controle, 2 casos $(1,29 \%)$.

Somente foram admitidas pacientes que utilizavam exclusivamente anticon. cepcional hormonal oral, não entrando nos grupos nenhuma associação com outro tipo de anticoncepcional.

O diagnóstico de gravidez foi feito por exame clínico e laboratorial sempre que necessário.

A Tabela I, mostra a distribuição por idade das pacientes

Algumas pacientes, como era de se esperar, apresentaram vários tipos de manifestações secundárias. Porém, as que eram de tal monta que levaram a suspensão do anticoncepcional, determinaram a exclusão do caso. Eram submetidas a controle médico cada seis meses

\section{TODOS}

o material obtido foi submetido a uma análise de variância segundo modelo $\mathrm{X}_{i j k}=\mu+A_{i}+\beta_{i j}+\xi_{i j k}$ que cor. responde a dois critérios de classifica. ção com hierarquia. Onde: $\mu=$ média geral; $A \mathbf{i}=$ efeito devido ao grupo $\mathbf{i} ; \beta_{i j}=$ Gfeito devido à idade $\mathrm{j}$ dentro do grupo i e $\xi=$ variação devida ao acaso. Os con-

TAB L L A 1

Distribuição por idade nos grupos

\begin{tabular}{r|r|r|c|c|c|c|c|c}
\hline Grupo & 19 & 20 & 21 & 22 & 23 & 24 & 25 & Total \\
\hline I & 21 & 23 & 24 & 27 & 20 & 26 & 21 & 162 \\
II & 15 & 19 & 21 & 21 & 18 & 16 & 17 & 127 \\
III & 9 & 13 & 15 & 18 & 21 & 21 & 24 & 121 \\
Controle & 21 & 22 & 22 & 25 & 21 & 23 & 21 & 155 \\
\hline Total & 66 & 77 & 82 & 91 & 80 & 86 & 83 & 565 \\
\hline
\end{tabular}


CIARI Jr., C. et al. - Relaçáo do tempo de uso de anticoncepcionais hormonais orais e tempo para conceber, Rev. Saúde publ., S. Paulo, 6: 273_81. 1972.

trastes lineares foram analisados pelo teste de Scherfé ${ }^{6}$.

\section{RESULTA DOS}

A distribuição do número de mulheres segundo tempo para conceber e tempo de uso de anticoncepcional hormonal oral consta da Tabela 2 e a distribuição de freqüências relativas é a constante da Tabela 3

TABELA 2

Distribuição das mulheres segundo tempo para conceber e tempo de uso de anticoncepcionais

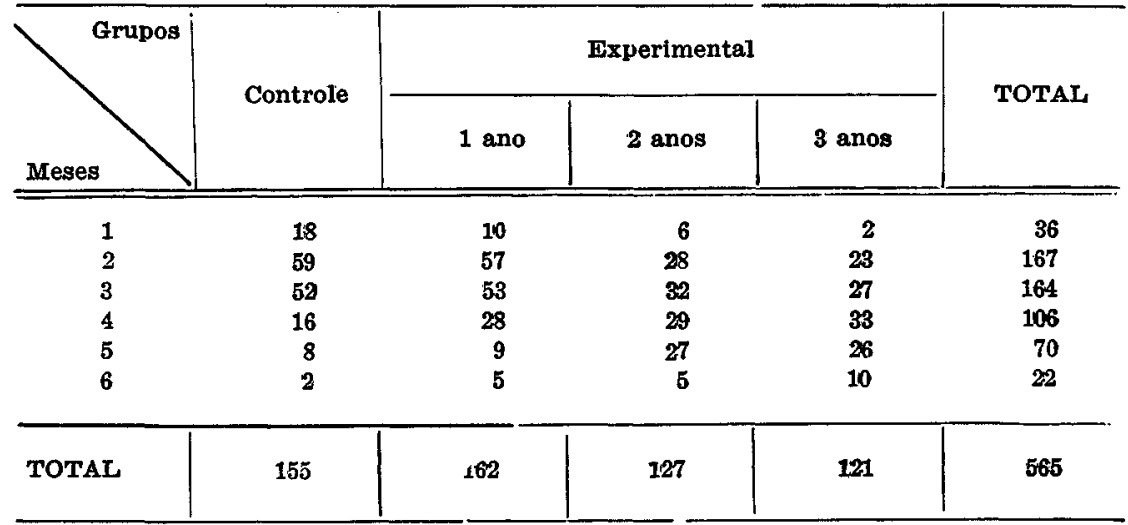

TABELA 3

Distribuição da frequência relativa das mulheres segundo tempo para conceber e segundo tempo de uso de anticoncepcionais

\begin{tabular}{c|c|c|c|c}
\hline \multirow{2}{*}{$\begin{array}{c}\text { Tempo para } \\
\text { conceber } \\
\text { (meses) }\end{array}$} & Tempo de uso de anticoncepcionais (anos) \\
& 0 & 1 & 2 & 3 \\
\hline \hline & & & & \\
\hline & 0,1161 & 0,0617 & 0,0472 & 0,0165 \\
3 & 0,3806 & 0,3519 & 0,2205 & 0,1901 \\
4 & 0,3355 & 0,3272 & 0,2520 & 0,2231 \\
5 & 0,1032 & 0,1728 & 0,2283 & 0,2727 \\
6 & 0,0516 & 0,0556 & 0,2126 & 0,2149 \\
& 0,0129 & 0,0509 & 0,0394 & 0,0826 \\
\hline
\end{tabular}

Da Tabela 4 constam as distribuições acumuladas de mulheres segundo o tempo para conceber e o tempo de uso dos anticoncepcionais.

Para cada um dos grupos calcularamse as médias, variâncias amostrais, bem como os desvios padrão e os coeficientes de variação. Os resultados constam da Tabela 5.
A título ilustrativo, fez-se constar da Figura 1 a distribuição de frequiência do tempo para conceber segundo o tempo de uso dos anticoncepcionais calculando-se nas abscissas a variável tempo e nas ordenadas as frequiências correspondentes.

A distribuição de frequiências acumuladas segundo tempo de uso de anticoncep- 
CIARI Jr., C. et al. - Relação do tempo de uso dẹ anticoncepcionais hormonais orais e tempo para conceber. Rev. Saúde públ., S. Paulo 6: 273-81 1972.

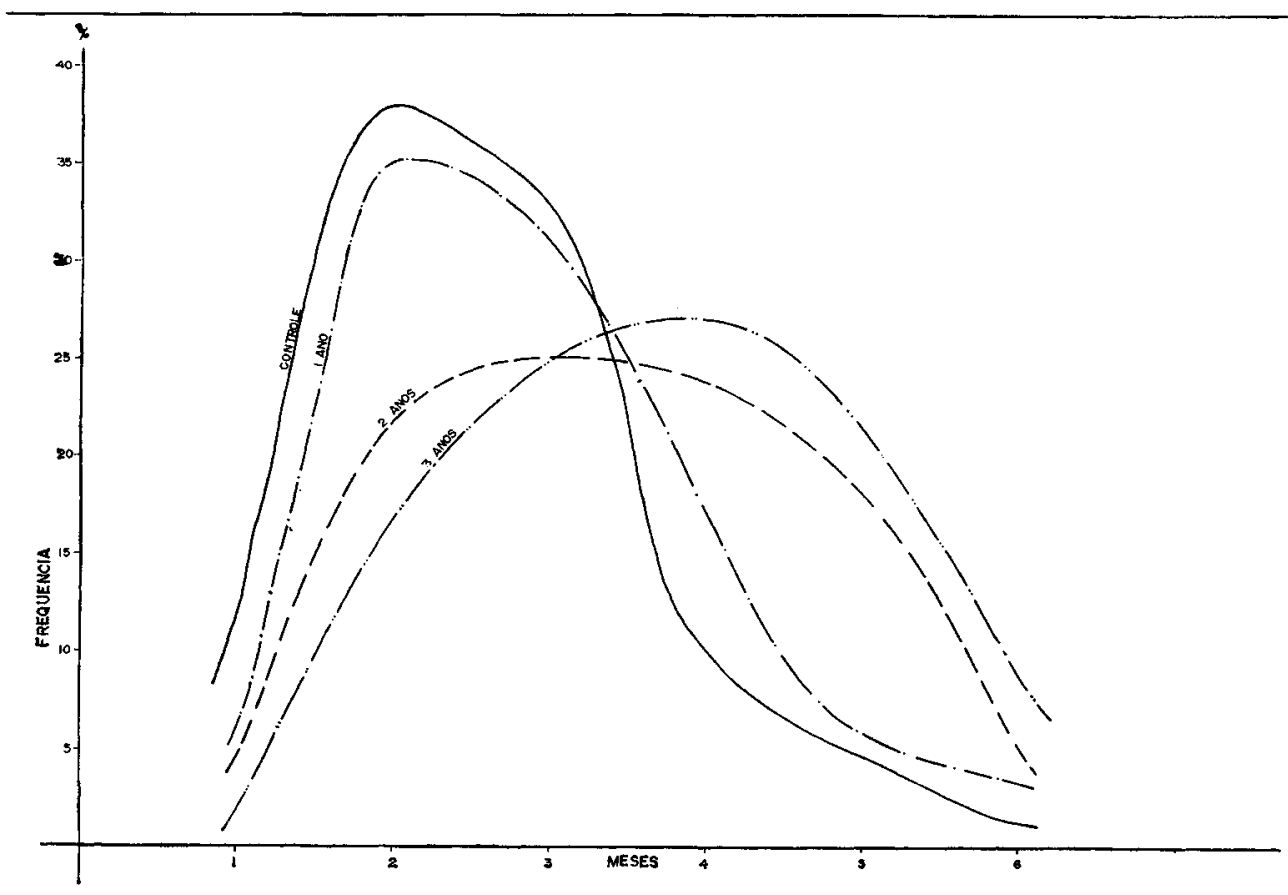

Fig. 1 - Distribuição de frequências do tempo para conceber, segundo tempo de uso das pílulas.

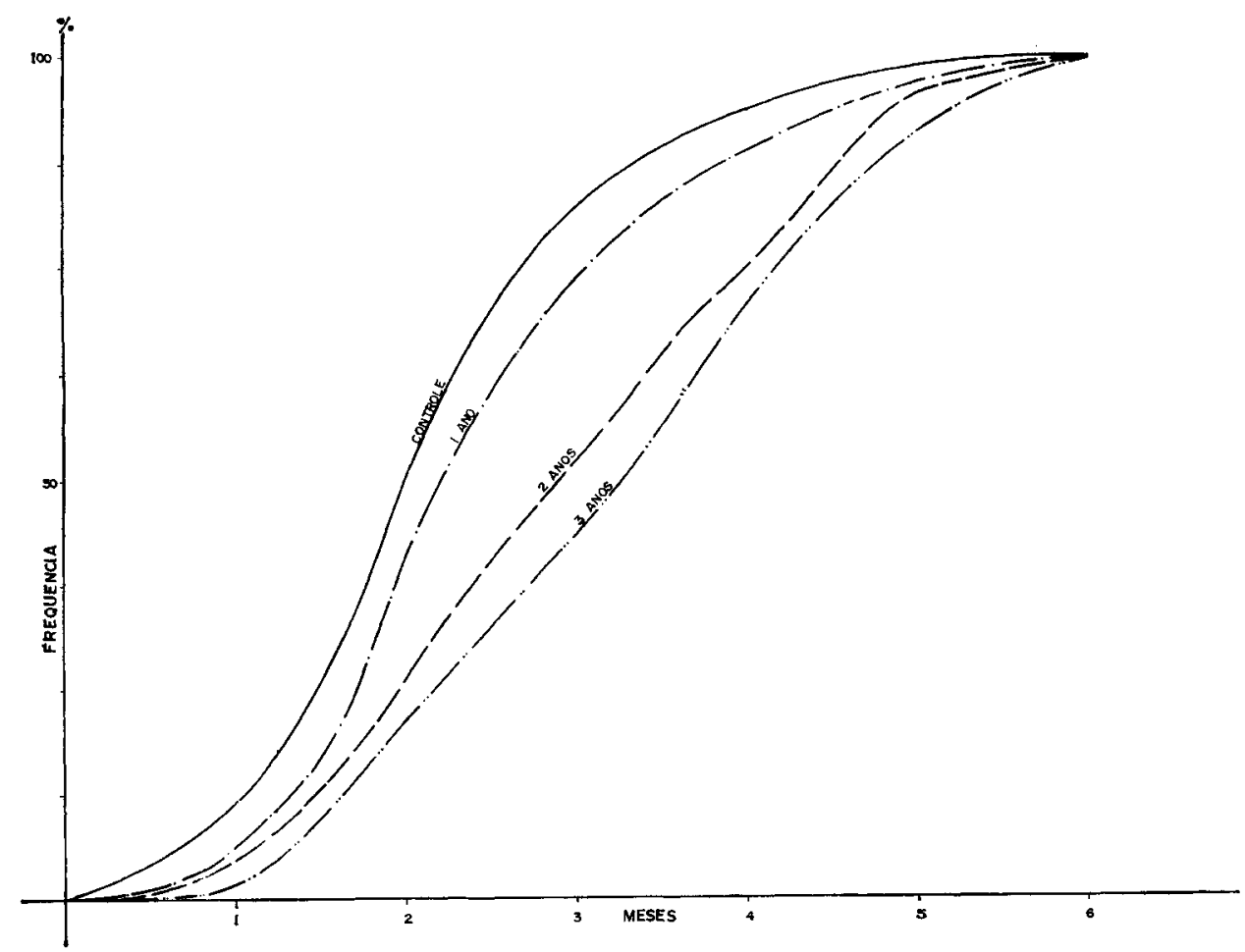

Fig. 2 - Distribuição acumulada de frequêencias do tempo para conceber, segundo tempo de uso de pílulas anticoncepcionais. 
CIARI Jr., C. et al. - Relaçăo do tempo de uso de anticoncepcionais hormonais orais e tempo para conceber. Rev. Saúde puibl., S. Paulo. 6: 273.81. 1072.

cionais, fez-se constar da Figura 2, onde novamente à abscissa corresponde a va- riável tempo e à ordenada a frequiência acumulada.

TA B L A 4

Distibuiçăo acumulada de mulheres segundo tempo para conceber e segundo tempo de uso de anticoncepcionais

\begin{tabular}{c|c|c|cc}
\hline $\begin{array}{c}\text { Tempo para } \\
\text { conceber } \\
\text { (meses) }\end{array}$ & 0 & Tempo de uso de anticoncepcionais (anos) & 3 \\
\hline \hline & 0,1161 & 1 & 2 & 0,0165 \\
2 & 0,49157 & 0,0617 & 0,0472 & 0,2066 \\
3 & 0,8322 & 0,4136 & 0,2677 & 0,4297 \\
4 & 0,9354 & 0,7408 & 0,5197 & 0,70424 \\
5 & 0,9870 & 0,9136 & 0,7480 & 0,9173 \\
6 & 1,0000 & 0,9692 & 1,0000 & 1,0000 \\
\hline
\end{tabular}

TA B F A 5

Distribuiçăo de média, variancia, desvio pađrăo e coeficiente de variaçăo nos grupos

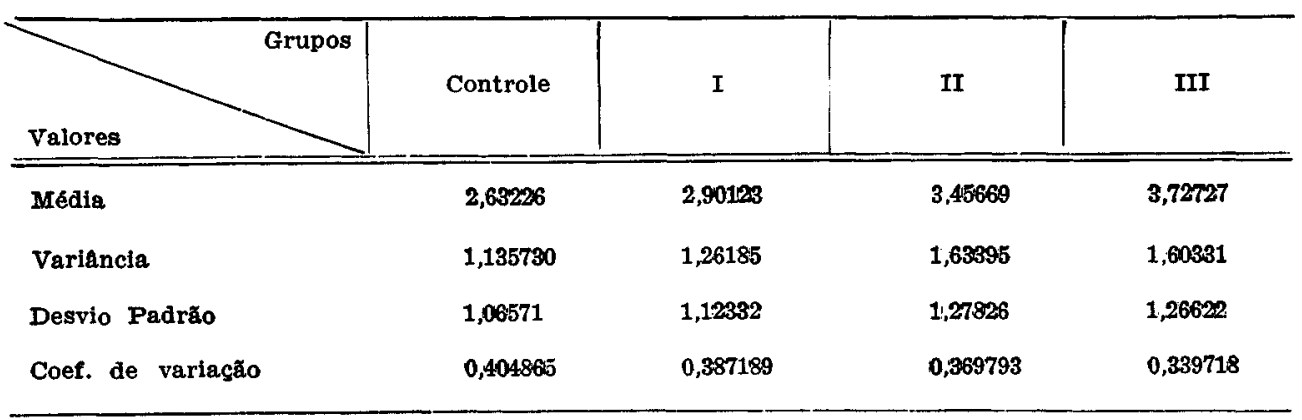

Ambas as Figuras sugerem claramente uma mudança de comportamento repro. dutivo quando o tempo de uso do anticoncepcional passa de 1 para 2 anos. Tanto na Figura 1 como na 2, as curvas correspondentes ao grupo controle e ao grupo I estão bem próximas. Contudo, distanciam-se apreciavelmente das curvas correspondentes aos grupos if e III que por sua vez mostram nítida aproximação.
Em face destes resultados considera. mos necessário submeter os dados obtidos à análise de variância segundo processo citado no capitulo de métodos.

Como resultado da análise de variância temos uma diferença estatisticamente significativa entre os grupos, ao nível de $1 \%$. Não houve diferenças quanto a idade dentro de cada grupo. (Tabela 6). 
CIARI Jr., C. et al. - Relação do tempo de uso de anticoncepcionais hormonais orais e tempo para conceber. Rev. Saúde públ., S. Paulo 6: 273.81. 1972.

TA B E A 6

Análise de variancia

\begin{tabular}{|c|c|c|c|c|}
\hline $\begin{array}{c}\text { Fontes de } \\
\text { variaçăo }\end{array}$ & g. $!$ & $\begin{array}{l}\text { Soma de } \\
\text { quadrados }\end{array}$ & $\begin{array}{l}\text { Quadrados } \\
\text { médios }\end{array}$ & $" F "$ \\
\hline Grupo & 3 & 103,5978 & 34,0326 & 13,03 * \\
\hline Idade (dentro de grupo) & 24 & 63,6208 & 2,6509 & - \\
\hline Residuo & ธ37 & 718,3495 & 1,3377 & 1,98 \\
\hline Total & 564 & 885,5681 & - & - \\
\hline
\end{tabular}

* Significante ao nivel de 0,01

TA B E A 7

Resultado das estatísticas de Scheffé

\begin{tabular}{l|l}
\hline Tipo de comparação & Estatística de Scheffé \\
\hline Grupo C x Grupo I & 2,08 \\
Grupo C x Grupo II & $5,86 *$ \\
Grupo C x Grupo III & $7,78 *$ \\
Grupo I x Grupo II & $3,93 *$ \\
Grupo I x Grupo III & $5,86 *$ \\
Grupo II x Grupo III & $1,8 \cap$ \\
\hline
\end{tabular}

Scheffé crítico ao nível de 0,01

$-3,38$

* Significante ao nivel de 0,01

Procurando saber se essas diferenças quanto a grupos eram devidas a contrastes lineares, aplicamos o teste de Scheffé cujos resultados constam da Tabela 7.

As diferenças entre médias de tempo para conceber foram, na maioria, esta. tisticamente significantes não havendo diferença apenas entre o grupo controle e o grupo I e o grupo II com o grupo III, confirmando o que foi sugerido pelas Fi. guras 1 e 2.

\section{DISCUSS A}

Pela análise estatística dos diferentes grupos, podemos considerá-los como equi- valentes quanto a idade. As mulheres foram selecionadas em serviços particulares das mesmas características e igualam-se sócio-economicamente bem como os exames clínicos as assemelham sob o ponto de vista de normalidade do aparelho genital e estado de saúde.

Desta forma, parece-nos que consegui. mos observar o comportamento do aparelho reprodutor após 1,2 e 3 anos de uso continuado de anticoncepcionais $e$ compará-lo a grupo semelhante sem esta prática.

$\mathrm{O}$ que observamos pela análise das Fi- 
CIARI Jr., C. et al. - Relação do tempo de uso de anticoncepcionais hormonais orais e tempo para conceber. Rev. Saúde puibl., s. Paulo. 6: 273-81. 1972.

guras é que comparado ao grupo controle, o grupo I parece responder ao uso de anticoncepcional após um ano de uma maneira semelhante, pois as curvas se aproximam (Figura 1). No entanto, nos grupos II e III revela-se uma nitida diferença de resposta do aparelho reprodutor ao uso continuado de anticoncepcio. nais hormonais orais depois de dois e três anos. Além disso, o que está ressaltado pelos gráficos, diante da proximida. de das curvas dos grupos controle e I por um lado e dos grupos II e III de outro, é que ultrapassando o tempo de um ano de uso a resposta muda sendo menos significativa quando vai de dois para três anos. Isto nos leva a supor que o momento crítico da mudança de comportamento do aparelho reprodutor se situa a partir de um ano de uso. Além disso, nota-se na Figura 1, com clareza, que a configuração das curvas dos grupos controle e I e dos grupos II e III sofrem nitida modificação o que caracteriza mais ainda a diferença de comportamento dos grupos. Seria possível ter-se visão melhor desta mudança se, a partir de um ano, os grupos fossem avaliados por período de tempo menor, ou seja, de seis meses. Além disso, acreditamos ser necessário observar-se os efeitos de uso em períodos mais prolongados (4 e 5 anos) se bem que seria difícil dada a raridade de casos de tomada por tanto tempo sem interrupģão ${ }^{5}$. Evidentemente que neste grupo dever-se-ia levar em consideração o natural declínio de fecundidade determinado pela idade, o que não ocorre significativamente em idades mais baixas.

i importante assinalar que estas tendencias apresentadas pelos grupos quanto à época de concepção apresentam uma pequena mobilidade, talvez devida a flutuações de amostras.

Sob o ponto de vista clínico, isto é, da forma de ação destes anticoncepcionais, se é feita sobre o ovário ou a hipófise, parece que afetando um destes componentes ou ambos, eles passam a responder deficientemente a partir deste período crítico. Indiscutivelmente, outros fatores podem ser determinantes desta protelação da gravidez, como alterações da mucosa uterina, $\mathrm{pH}$ vaginal, motilidade tubária, etc. que merecem ser estudados separadamente. GoldzirHer et al. 2, 1962, procuraram observar o efeito do anticon cepcional hormonal usado durante um e até dois anos. O controle foi feito por biopsia do endométrio revelando poucas alteraçōes nos primeiros doze meses e maiores nos demais. Em 41 mulheres que interromperam 0 anticoncepcional para engravidar, $62 \%$ tiveram sucesso no primeiro mês. No entanto, não especificam o comportamento do restante do grupo.

O que fica evidenciado é que, seja qual for o modo de ação do anticoncepcional, o seu uso continuado a partir de dois anos revela uma alteração da resposta do aparelho reprodutor quanto ao tempo para engravidar.

\section{CONCLUSOES}

1. Pela análise dos resultados verificase que o tempo para concepção das muIneres que tomaram 0 anticoncepcional hormonal oral durante um ano é igual ao do grupo controle, como é sugerido pelas Figuras 1 e 2.

2. As mulheres que tomaram o mesmo anti-concepcional durante dois e três anos tiveram tempo para conceber bastante maior.

3. Isto nos leva a supor uma interferência do anticoncepcional no processo reprodutivo já que os grupos são comparáveis e foram respeitadas as variáveis propostas por CoRfMAN 1 . 
CIARI, Jr., C. et al. - Relação do tempo de uso de anticoncepcionais hormonais orais e tempo para conceber. Rev. Saúde puibl., S. Paulo 6: 573-81 1972.

RSPSP-141

CIARI $\mathrm{J}_{\mathrm{R} .}, \mathrm{C}$. - - The required for concepcion: it's relation to the oral hormonal contraceptive]. Rev. Saúde públ., S. Paulo, 6: 273-81, 1972.

Summary: A total of 565 women was partioned in four groups: the control group with 155 women, who started the sexual life without any contraceptive control; group 1 , with 162 women who had been taking oral contraceptive auring a period of one year, starting at the beginning of their sexual lifes; group II, with 127 women who had been taking the same oral contraceptive during a period of 2 years starting at the beginning of their sexual lifes; group III, with 121 women who repeated the same procedure during a period of 3 years. All the 565 women had the same social economic status and the same health conditions; were between 19 and 25 years old and used the contraceptive in a same way. The frequency distributions of time required for conception showed a clear difference between groups control and I as compared to groups II and III. The analysis of variance confirmed the difference. Based on these results, it is con. cluded that oral hormonal contraceptive after the second year of continous use interfer with the reproductive system, this determining a larger time required for conception.

UnITERMS: Fertility *; Contraceptive. oral.

\section{REFERENCIAS BIBLIOGRAFICAS}

1. CORFMAN, P. A. - Oral contraceptive: 2nd report. New York, Food and Drug Administration. Adisory Committe on Obstetrics \& Gynecology, 1969. p. 71 .

2. GOLDZIEHER, Y. W. et al, - Study of norethindrone in contraception. JAMA, 180: 359-61, 1962 .

3. GOLDZIEHER, $\mathbf{Y}$. W, et al. - Fertility following termination of contraception with norethindrone, Amer. J. Obst. Gynec. 84: 1474-77, 1962.

4. PINCUS, G. - Suppression of ovulation with reference to oral contraceptive. In: MODERN trends in endocrinology: 2nd serie. London, Butterworths, 1961. p. 231.45.

5. RYDER, N. B. \& WESTOFF, C. F. - Use of oral contraceptive in USA. Science, 153: 1199-205, 1966.

6. SCHEFFr, R. - A method for judging all contraste in the analysis of variance. Biometrikes, 40: 87-104, 1953.

Recebido para publicaço em 14.7-1972

Aprovado para publicardo em 26.7-1972 